SMAD, Rev. Eletrônica Saúde Mental Álcool Drog.

2019 abr.-jun.;15(2):4-11

DOI: 10.11606/issn. 1806-6976.smad.2019.152345

www.revistas.usp.br/smad/

Artigo Original

\title{
Uso de álcool e determinantes psicossociais entre estudantes de medicina e tecnologia médica
}

Maria Josefa Arcaya Moncada ${ }^{1}$

Daysi Coras Bendezu²

Sandra Cristina Pillon ${ }^{3}$

\footnotetext{
* Apoio financeiro da Secretaria Nacional de Políticas sobre Drogas, Comissão Interamericana de Controle do Abuso de Drogas (SENAD/CICAD), Brasil e da Universidad Nacional Mayor de San Marcos, Peru.

${ }^{1}$ Universidad Nacional Mayor de San Marcos, Lima, Peru.

${ }^{2}$ Instituto Nacional de Salud del Niño San Borja, Lima, Peru.

${ }^{3}$ Universidade de São Paulo, Escola de Enfermagem de Ribeirão Preto, Centro Colaborador da OPAS/OMS para o Desenvolvimento da Pesquisa em Enfermagem, Ribeirão Preto, SP, Brasil.
}

O estudo teve por objetivo avaliar o uso de álcool e as possíveis associações com os determinantes psicossociais em estudantes de medicina e tecnologia médica da Faculdade de Medicina da Universidade de San Marcos, Peru. O estudo é do tipo transversal da abordagem quantitativa realizados com 219 estudantes. O Teste de identificação do uso de álcool e os determinantes psicossociais relacionados ao álcool foram utilizados. A prevalência de uso de álcool no último ano foi de $69,5 \%$, com predominância de uso pelo sexo masculino $(71,6 \%)$, e jovens (79,9\%). As associações foram entre o uso de álcool e o estresse, com predominância da sobrecarga acadêmica, esses dados são relevantes para o planejamento de ações preventivas do uso de álcool nessa população.

Descritores: Transtornos Relacionados ao Uso de Álcool; Determinantes Sociais da Saúde; Educação; Graduação em Medicina.

\section{Como citar este artigo}

Moncada MJA, Bendezu DC, Pillon SC. Consumption of Alcohol and Psychosocial Determinants among Students of Medicine and Medical Technology. SMAD, Rev Eletrônica Saúde Mental Álcool Drog. 2019;15(2):4-11. doi: https://dx.doi.org/10.11606/issn.1806-6976.smad.2019.152345 


\title{
Consumption of Alcohol and Psychosocial Determinants among Students of Medicine and Medical Technology
}

\begin{abstract}
The objective was to evaluate alcohol consumption and its association with psychosocial determinants among students of medicine and medical technology at the Faculty of Medicine, in the Universidad Nacional Mayor de San Marcos, Peru. This cross-sectional study was designed with 219 students. The Alcohol Use Disorder Identification Test and psychosocial determinants were used. The prevalence of alcohol use in the last year was $69.5 \%$, with predominance of use by male (71.6\%) and young students (79.9\%). Associations were observed between the use of alcohol and stress, with predominance of academic overload. The results provide information relevant to the planning of preventive actions of alcohol use for this population.
\end{abstract}

Descriptors: Alcohol-related Disorders; Psychosocial Determinants; Education; Undergraduate in Medical; Social Determinants of Health.

\section{Consumo de alcohol y determinantes psico-sociales en estudiantes de medicina y tecnología médica}

\begin{abstract}
El estudio tuvo por objetivo evaluar el consumo de alcohol y con los determinantes psicosociales en estudiantes de medicina y tecnología médica de la Facultad de Medicina de la Universidad de San Marcos, Perú. Estudio del tipo transversal, de abordaje cuantitativo realizado con 219 estudiantes. El test de identificación del uso de alcohol y los determinantes psicosociales relacionados al alcohol fueron utilizados. La prevalencia de consumo en el último año fue de $69.5 \%$, con predominancia de uso entre el sexo masculino (71,6\%) y jóvenes (79,9\%). Las asociaciones fueron entre el uso de alcohol y estrese, predominando la sobre carga académica, eses datos son relevantes para el planeamiento de acciones preventivas frente al uso de alcohol en esa población.

Descriptores: Trastornos Relacionados con Alcohol; Determinantes Sociales de la Salud; Educación; Pregrado en Medicina.
\end{abstract}




\section{Introdução}

O consumo de bebidas alcoólicas é um importante fator de risco, resulta em altas taxas de mortalidade e morbidade em diversos países, especialmente na América Latina $^{(1-5)}$. Estudos mostram taxas mais elevadas do uso de álcool e de drogas, com consequências negativas entre os mais jovens (18 a 25 anos de idade), dos quais, uma grande parte são estudantes universitários. Dados do II Estudo Epidemiológico Andino sobre o uso de drogas na população universitária(5) mostraram que o álcool é a droga mais comumente consumida (71,9\%) entre os estudantes e estimaram que cerca de $8 \%$ dos estudantes peruanos apresentaram possíveis sinais de dependência do álcool.

Estes números foram maiores somente entre os alunos que relataram o uso de álcool pelo menos uma vez no último ano, especialmente, os homens mais jovens. A percepção de danos ou riscos e o consumo de álcool foi maior em quatro países (75\%), a prevalência de consumo entre homens foi de $32,5 \%$ no Peru e na Bolívia 48,3\%, 35,5\% na Colômbia e 43,9\% no Equador. Entre as mulheres, por outro lado, as taxas foram de $29,7 \%$ na Bolívia, $27,1 \%$ na Colômbia, 28,8\% no Equador e $17,7 \%$ no Peru(3).

Ao comparar os estudos realizados com universitários da Comunidade Andina (CAN) de 2009 e 2012, observa-se incremento significativo do consumo de álcool no último ano (64,4\% em 2009 e 71,7\% em $2012)$, tanto entre os homens $(72,5 \%$ e $78,3 \%)$ como entre as mulheres $(57,4 \%$ e $65,9 \%)$. Também pode ser observado incremento do consumo em nível de risco ou prejudicial de álcool, 29,5\% em 2009 e 31,9\% em 2012, com poucas variações desse consumo entre as mulheres em 2009 (15,9\%) e 2012 (18\%).

As percentagens de estudantes universitários com sinais de dependência do álcool também aumentaram significativamente, de 8,3\% em 2009 para 10,8\% em 2012, levando à conclusão de que se trata de um problema de saúde altamente preocupante, que gera impacto nas taxas de desempenho e abandono $\operatorname{acadêmico}^{(3-4)}$.

Em 2010, a Comissão Nacional para o Desenvolvimento e Vida sem Drogas (DEVIDA) no Peru, avaliou a prevalência de uso na vida de drogas na população geral (12 a 65 anos) mostrou o número de casos de alcoolismo no período de 12 anos (1998 a 2010) e estimou que há uma tendência decrescente no consumo de álcool, mais evidente a partir de 2006, de $83.0 \%$ para $75,4 \%$ em 2010 . A incidência anual de estudantes que começaram a consumir álcool em 2006 foi de $30,2 \%$ e $26,4 \%$ em $2010^{(5)}$.

As condições sociais que os alunos vivem e trabalham podem fortemente influenciar o consumo de álcool. Atualmente, o estilo de vida, o ambiente, bem como biologia humana e a organização de serviços de saúde são reconhecidos como os principais determinantes de saúde dos indivíduos, segundo a Organização Mundial da Saúde (OMS), embora esses tenham sido mais estudado na Europa e Estados Unidos (EUA) e muito pouco em países da América Latina(6).

No contexto microssocial ao entorno do estudante de universidade pública, existem condições sociais que incentivam o consumo de álcool. Considerando que a maioria deles são de classes socioeconômica mais baixa, de famílias migrantes da região dos Andes, com cultura, hábitos e estilos de vida que aprenderam com o seu ambiente familiar por meio de modelos parentais, influenciando o hábito de consumo de álcool. Um estudo mostrou que para $84,4 \%$ dos estudantes de Odontologia, o curso é estressante e entre os elementos considerados estressantes é ter que apresentar um determinado número de trabalhos clínicos durante um período curto de tempo, bem como atender pacientes não cooperativos com tratamento odontológico(7).

Em Lima, a prevalência do uso de álcool entre adolescentes foi de $42,2 \%$ e o fator psicológico mais fortemente associado ao uso de drogas foi o estresse psicológico severo(8).

Evidência sugere que entre os principais determinantes psicossociais do uso da substância entre os jovens são as formas que eles lidam com o estresse em suas vidas, as expectativas dos efeitos da substância, pressão dos pares e normas percebidas, bem como a autoeficácia em recusar as substâncias que Ihes são oferecidas. Os resultados de estudos sobre o estresse acadêmico vão desde o reconhecimento à afirmação da relação direta existente entre essas variáveis, permitindo assim afirmar que os estudantes mais estressados são os que tiraram as melhores notas ${ }^{(9)}$.

Esta situação foi evidenciada em estudantes de medicina, pela a presença de estressores acadêmicos tais como competitividade entre o grupo de pares, exames, sobrecarga de tarefas, apresentações, problemas relacionados com professores e colegas, um ambiente social desagradável e tempo limitado, entre outros fatores que provocam situações estressantes, produzindo mecanismo de enfrentamento inadequado sob pressão para ser parte de um grupo.

Além disso, o consumo de álcool em pequena ou grande quantidade é frequentemente observado em celebrações ou eventos sociais no âmbito universitário, provocando euforia e imprudência que pode resultar em comportamentos com consequências negativas.

Existe ainda evidências na América Latina que mostram resultados similares, em países com algumas variações, como no Peru, onde o consumo do álcool tende a diminuir nos últimos anos(10).

Por outro lado, existem poucas evidências de estudos em países da América Latina que abordam 
os fatores psicossociais relacionados ao consumo de álcool na população universitária, não existem estudos até o momento sobre a perspectiva dos determinantes psicossociais.

O presente estudo é baseado na teoria de Determinantes Sociais da Saúde (DSS), entendido como as condições sociais em que os estudantes universitários vivem, estudam e trabalham. Este modelo propõe que os indivíduos e os seus comportamentos são influenciados por sua comunidade e redes sociais que podem apoiar ou não a saúde dos sujeitos. A estratificação socioeconômica pode configurar em melhores ou piores oportunidades para a saúde. Ele não age diretamente, mas é mediada por uma variedade de fatores: habitação, circunstâncias psicossociais e comportamentos (hábito de fumar, uso de álcool ou má alimentação) ${ }^{(11)}$.

O consumo de álcool é definido por uma série de fatores interligados, tais como: características individuais, características do entorno microssocial imediato (família, amigos, trabalho e comunidade) e fatores macrossociais (sistema social, cultura de valores imperante, meios de comunicação e a geografia física) que os indivíduos desenvolvem os seus comportamento ${ }^{(11)}$.

Os principais determinantes para o consumo de álcool entre os jovens são a família e a cultura geral do consumo. Amigos e colegas são de particular importância, dada sua influência social e suas relações.

Fatores envolvidos na determinação das decisões em relação ao consumo de álcool, os padrões de consumo e a probabilidade de resultados benéficos ou prejudiciais podem ser agrupadas em quatro grandes categorias: predisposição genética, características individuais, fatores socioeconômicos e determinantes ambientais. O consumo abusivo de álcool e a disfunção familiar são fatores que influenciam o consumo de outras substâncias psicoativas em estudantes universitários ${ }^{(12)}$.

Um outro fator relevante ao consumo de álcool é o estresse acadêmico. Entrar em uma instituição de ensino, manter-se como aluno regular e graduar-se são consideradas experiências estressantes por muitos estudantes. $O$ estresse acadêmico envolve diferentes aspectos, tais como: a vulnerabilidade ao estresse, $69 \%$ dos estudantes de medicina podem ser considerados vulneráveis ao estresse. No Chile, a prevalência do estresse é de $36,3 \%$, maior entre as mulheres $(p<0.05)$, o curso de medicina é a carreira mais estressante que os demais cursos. O primeiro ano do curso de Medicina é o mais estressante que os demais anos da graduação $(p<0,01)^{(13)}$.

No Peru, elevados níveis de estresse foram observados em estudantes de medicina, com prevalência de uso na vida, uso e abuso de álcool, cigarros; comportamentos violentos e antissocial, além de hábitos de vida afetados pelas exigências do curso de medicina e poucas oportunidades de diversão social ou de entretenimento(10).

Em estudantes de enfermagem, a sobrecarga acadêmica foi o estressor mais prevalecente ${ }^{(14)}$. Mediante esta situação, há uma necessidade justificável para estudar o consumo de álcool em estudantes de Medicina e Tecnologia Médica na perspectiva dos determinantes da saúde.

O objetivo deste estudo foi avaliar o uso de álcool entre estudantes de Medicina e Tecnologia Médica e as relações possíveis com os determinantes psicossociais.

\section{Método}

Este é um estudo descritivo transversal da abordagem quantitativa. O estudo foi realizado na Escola de Medicina e Tecnologia Médica da Universidade Nacional Mayor de San Marcos em Lima, Peru.

Os critérios de elegibilidade foram: ter idade igual ou maior que 18 anos, estar matriculado no curso de graduação em Medicina e a Tecnologia Médica. Os alunos que estavam afastados por licença médica ou ausentes por três dias consecutivos nas aulas, durante o período de coleta de dados foram excluídos.

Do total de 1192 estudantes matriculados, 219 estudantes foram selecionados, pelo método de amostragem aleatória simples, dos quais correspondem a $127(58 \%)$ de medicina y $92(42 \%)$ de tecnologia médica.

Um questionário foi composto pelo Teste de identificação dos problemas relacionados ao uso de álcool (AUDIT) e a avalição dos determinantes psicossociais do consumo de álcool foi utilizado. O AUDIT foi desenvolvido pela $\mathrm{OMS}^{(15,16)}$, tem por objetivo identificar os níveis de risco de uso do álcool e realizar uma intervenção breve em relação ao consumo de álcool. Esse teste é composto por 10 itens. Ele foi validado nos EUA antes de ser culturalmente adaptado e validado para uso em espanhol em vários países da América Latina, incluindo o Peru. A sensibilidade do AUDIT é de 57-59\% e especificidade de $91-96 \%{ }^{(16)}$.

O questionário de avaliação dos determinantes psicossociais está composto por 30 itens que avaliam a presença ou ausência de determinantes psicossociais específicos. O instrumento foi submetido a validação semântica. Informações foram processadas na tabela de concordância e teste binomial. A validade foi realizada mediante o teste de coeficiente de correlação de Pearson (os itens são válidos para os valores de $r>0,2$ ), valores de $p$ binomial de 0,036 e o grau de concordância $p<0,05$. $\mathrm{O}$ teste de confiabilidade foi realizado por meio do Alfa de Cronbach de>0,7. Um teste piloto foi realizado com 20 estudantes, o instrumento é confiável para sua utilização, com valor de Alfa de Cronbach=0,951. 
Os diretores da Faculdade de Medicina da Universidade Nacional Mayor de San Marcos foram formalmente solicitados para autorizar o estudo. Os dados foram coletados no final da aula, em colaboração com o professor. Os alunos que voluntariamente concordaram em participar do estudo, em seguida, assinaram o Termo de Consentimento Livre Esclarecido.

O projeto foi submetido e aprovado pelo Comitê de Ética em Pesquisa da Universidad Nacional Mayor de San Marcos, Processo número 314, em conformidade com normas peruanas para pesquisas envolvendo seres humanos, garantindo o anonimato, respeitando a privacidade e com possibilidade de desistência do participante em qualquer etapa da pesquisa. Os dados foram coletados no segundo semestre de 2016.

Os dados foram analisados utilizando o Statistical Package for Social Sciences (SPSS) versão 17.0. Para análise estatística foi utilizado o teste de Chi-quadrado e o Exato de Fisher, que permitem avaliar as associações entre as variáveis socioeconómicas e uso de álcool. Para as variáveis categóricas foram calculadas as frequências absoluta (N) e relativa (\%). As variáveis continuas foram descritas em termos de valores médio e desvio-padrão. O nível de significância de 5\% foi adotado para todos os testes.

\section{Resultados}

A Tabela 1 mostra os dados sociodemográficos e o uso de álcool no último ano. A amostra foi composta por 219 estudantes da medicina e tecnologia médica, caracterizados pelo sexo masculino 119 (58,9\%), idade entre 21 e 25 anos de idade 132 (65\%), solteiros 201 (99\%), provenientes da região da Costa, 173 (85,3\%) e viviam com algum familiar $165(81,7 \%)$.

Do total de estudantes, $141(69,5 \%)$ consumiram bebidas alcoólicas no último ano. Na amostra as diferenças foram entre consumir bebidas alcoólicas, sexo (masculino $71,6 \%$ versus $28,4 \%$ feminino, $p \leq 0,001$ ) e faixa etária ( $21-25$ anos $79,9 \%$ versus $14,2 \% \geq 26$ anos, $p=0,011)$.

Tabela 1 - Informações sociodemográficas e uso de álcool no último ano, entre estudantes de medicina e tecnologia médica. Universidade Nacional Mayor de San Marcos. Lima, Perú, 2015. N=219

\begin{tabular}{|c|c|c|c|c|c|}
\hline & & \multirow{2}{*}{ Total } & \multicolumn{2}{|c|}{ Consumo de Álcool } & \multirow{3}{*}{$\begin{array}{l}\text { Valor } \\
\text { de } p\end{array}$} \\
\hline & & & Sim & Não & \\
\hline & & $f^{*}(\%)$ & $f^{*}(\%)$ & $f^{*}(\%)$ & \\
\hline \multirow{2}{*}{ Sexo } & Feminino & $84(41,4)$ & $40(28,4)$ & $44(71,0)$ & \multirow{2}{*}{$<0,001^{\text {tf }}$} \\
\hline & Masculino & $119(58,6)$ & $101(71,6)$ & $18(29,0)$ & \\
\hline \multirow{3}{*}{$\begin{array}{l}\text { Faixa } \\
\text { etária } \\
\text { (anos) }\end{array}$} & $\leq 20$ & $41(20,2)$ & $21(14,9)$ & $20(32,3)$ & \multirow{3}{*}{$0,011^{\S^{\ddagger}}$} \\
\hline & $21-25$ & $132(65,0)$ & $100(70,9)$ & $32(51,6)$ & \\
\hline & $\geq 26$ & $30(14,8)$ & $20(14,2)$ & $10(16,1)$ & \\
\hline \multirow{2}{*}{$\begin{array}{l}\text { Estado } \\
\text { civil }\end{array}$} & Solteiros & $201(99,0)$ & $141(100,0)$ & $60(96,8)$ & \multirow{2}{*}{$0,092^{+}$} \\
\hline & Casado & $2(1,0)$ & $2(3,2)$ & $-(-)$ & \\
\hline
\end{tabular}

(continua...)

\begin{tabular}{|c|c|c|c|c|c|}
\hline & \multirow{3}{*}{$\begin{array}{l}\text { Total } \\
f^{*}(\%)\end{array}$} & \multicolumn{2}{|c|}{ Consumo de Álcool } & \multirow{3}{*}{$\begin{array}{l}\text { Valor } \\
\text { de } p\end{array}$} \\
\hline & & & \multirow{2}{*}{$\frac{\operatorname{Sim}}{f^{\star}(\%)}$} & \multirow{2}{*}{$\begin{array}{c}\text { Não } \\
f^{*}(\%)\end{array}$} & \\
\hline & & & & & \\
\hline \multirow{3}{*}{ Origem } & Costa & $173(85,2)$ & $121(85,8)$ & $52(83,9)$ & \multirow{3}{*}{$0,771^{\S}$} \\
\hline & Montanha & $23(11,3)$ & $16(11,3)$ & $7(11,3)$ & \\
\hline & Floresta & $7(3,4)$ & $4(2,8)$ & $3(4,8)$ & \\
\hline \multirow{3}{*}{$\begin{array}{l}\text { Com } \\
\text { quem } \\
\text { vive }\end{array}$} & $\begin{array}{l}\text { Membro da } \\
\text { família }\end{array}$ & $165(81,7$ & $113(80,7)$ & $52(83,9)$ & \multirow{3}{*}{$0,625^{\S}$} \\
\hline & Sozinho & $18(8,9)$ & $12(8,6)$ & $6(9,7)$ & \\
\hline & $\begin{array}{l}\text { Amigos/ } \\
\text { outros }\end{array}$ & $19(9,4)$ & $15(10,7)$ & $4(6,5)$ & \\
\hline
\end{tabular}

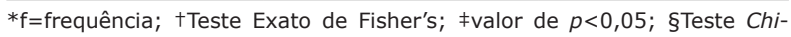
quadrado

A Tabela 2, mostra dados sobre os determinantes sociais e uso de álcool. Na amostra total, observa-se um predomínio de ausência de problemas em relação aos aspectos família $(63,3 \%)$ e amigos $(62,1 \%)$.

Entre os estudantes, os tipos de problemas predominantes foram relacionados ao trabalho $(56,2 \%)$, estudo $(65 \%)$, estressores $(72,4 \%)$ e pressão para consumir bebidas alcoólicas (77,8\%). Destaca-se que entre os estudantes com a presença de estressores era maior entre os que não consumiam bebidas alcoólicas $(83,9 \%)$ quando comparados aos que consumiam $(67,4 \%)$, com valores estatísticos significativos $(p=0,011)$

Tabela 2 - Determinantes sociais e uso de álcool no último ano passado, segundo os estudantes de medicina e tecnologia médica da Universidad Nacional Mayor de San Marcos. Lima, Perú, 2015. N=219

\begin{tabular}{cccccc} 
& & Total & \multicolumn{2}{c}{ Consumo de Álcool } & \multirow{2}{*}{ Valor } \\
\cline { 3 - 5 } & & & Não & Sim & de $\boldsymbol{p}$ \\
\cline { 3 - 5 } & & $\mathbf{f}^{*}(\%)$ & $\mathbf{f}^{*}(\%)$ & $\mathbf{f}^{*}(\%)$ & \\
\hline \multirow{2}{*}{ Família } & Absent & $129(63,5)$ & $43(69,4)$ & $86(61,0)$ & \multirow{2}{*}{$0,163^{+}$} \\
& Present & $74(36,5)$ & $19(30,6)$ & $55(39,0)$ & \\
Amigos & Absent & $126(62,1)$ & $36(58,1)$ & $90(63,8)$ & \multirow{2}{*}{$0,266^{+}$} \\
& Present & $77(37,9)$ & $26(41,9)$ & $51(36,2)$ & \\
Trabalho & Absent & $89(43,8)$ & $33(53,2)$ & $56(39,7)$ & \multirow{2}{*}{$0,051^{+}$} \\
& Present & $114(56,2)$ & $29(46,8)$ & $85(60,3)$ & \\
Estudo & Absent & $71(35,0)$ & $24(38,7)$ & $47(33,3)$ & \multirow{2}{*}{$0,280^{+}$} \\
& Present & $132(65,0)$ & $38(61,3)$ & $94(66,7)$ & \\
Estressores & Absent & $56(27,6)$ & $10(16,1)$ & $46(32,6)$ & \multirow{2}{*}{$0,011^{\ddagger 5}$} \\
\cline { 4 - 5 } $\begin{array}{c}\text { Pressão } \\
\text { para o uso } \\
\text { de álcool }\end{array}$ & Present & $147(72,4)$ & $52(83,9)$ & $95(67,4)$ & \\
\hline
\end{tabular}

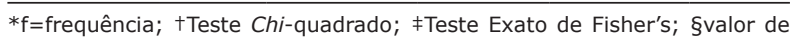
$p<0,05$

\section{Discussão}

O presente estudo buscou uma melhor compreensão entre o uso de álcool e as possíveis associações com os determinantes psicossociais em uma amostra de estudantes peruanos de medicina e tecnologia médica.

A prevalência do consumo de álcool no último ano pelos estudantes foi de $69,5 \%$. O álcool é a substância que está muito presente na vida dos estudantes. Estes dados contrastam com a prevalência do uso de álcool em 
estudantes de medicina e de outras áreas da saúde ${ }^{(17-18)}$, bem como da população peruana, que são bastante superiores.

Nos achados do estudo(10) as prevalências de uso de álcool na vida entre estudantes de duas escolas peruanas de medicina foram de $95,6 \%$ e $89,8 \%$ respectivamente. Os índices são bem baixo quando se compara com os jovens da população geral peruana, a prevalência de uso de álcool na vida foi $61 \%$ entre os mais jovens (15 a 17 anos) e $89 \%$ entre os de 21 a 24 anos, a idade de início do uso de álcool entre os estudantes foi de 15 anos $^{(10)}$.

Outro estudo com estudantes mexicanos de medicina, a prevalência de uso de álcool na vida foi de $71,9 \%$ e a idade de início 12,5 anos. A frequência de consumo foi baixa, mas em alta quantidade e o consumo em níveis de embriaguez, principalmente entre os homens. Nessa amostra foi avaliado o uso de drogas (licitas e ilícitas) e a prevalência de uso de álcool foi de $52,6 \%$, níveis semelhantes ao de outros países ${ }^{(18)}$.

Entre os estudantes universitários colombianos, a prevalência de consumo de álcool tem sido muito elevada quando comparada a do presente estudo, foi observado que nove a cada dez estudantes consumiam bebidas alcoólicas ${ }^{(19)}$. Um estudo em Honduras, reportou que somente $25 \%$ dos estudantes de medicina haviam consumido bebidas alcoólicas recreacionalmente(20).

Em relação aos determinantes psicossociais (Tabela2), no presente estudo não foi encontrado diferenças entre o uso de álcool e os determinantes relacionados à família e aos amigos. Esse resultado sugere que a integração, o suporte familiar e o de amigos exercem um papel determinante e favorável, por se constituir entre os estudantes um apoio essencial frente ao consumo de álcool. Uma outra justificativa para este achado, pode estar relacionado as questões familiares, uma vez que a maioria dos estudantes vivem com alguém da família e provavelmente recebem suporte, ambiente e comunicação, relacionamentos, papel do modelo, amor percebido da família, esses são elementos que podem contribuir para diminuir as possibilidades de consumo.

O entorno familiar, os amigos e as redes de apoio social influenciam o desenvolvimento de padrões e a trajetória de consumo de álcool do indivíduo ao longo do tempo. A influência familiar persiste na vida adulta, o que pode ser um fator de enfrentamento do uso problemático(21-22).

As pessoas com laços familiares mais fortes tendem ser menos influenciadas pelos amigos e com poucas habilidades para evitar o consumo de risco de álcool. Dessa maneira, melhores estreitamentos dos laços familiares, somados a maior participação nas atividades familiares caracterizam-se por ser importantes fatores de proteção(22).
Em nosso estudo, por outro lado, foi observado a existência de problemas relacionados ao trabalho (satisfação com o trabalho, salário e relacionamento), estudos (desempenho) e pressão para o consumo do álcool (celebração social e normas permissivas). Isso indica que os estudantes com tais características estão mais expostos aos riscos, criado pela exposição as condições laborais estressantes, estudo, pressão e a presença de estressores relacionados ao consumo de álcool.

O emprego, a qualidade do ambiente de trabalho, a segurança física, mental e social nas atividades laborais, incluindo a capacidade de controle sobre as demandas e pressão de trabalho são importantes determinantes a saúde. A igualdade de acesso à educação, a qualidade da educação recebida e a oportunidade de colocar em prática as habilidades aprendidas também são fatores muito importantes nas condições de vida e estado de saúde da população(23).

Um dos principais resultados deste estudo, foi a associação positiva entre uso de álcool e estresse, o que refere a capacidade de resolver situações problemáticas, exames (provas de avaliação cognitiva) e tarefa, a relação com professores e profissionais de saúde. Existem mais alunos com problemas relacionados com o estresse entre aqueles que não bebem, em comparação com aqueles que consomem bebidas alcoólicas. Este fato pode ser explicado pela adoção de outros estilos de enfretamento negativo, como fumar, comer demais ou negação do problema.

Estudos sobre estressores se dividem em duas categorias: os que abordam os estressores de forma genérica, incluindo os académicos e os que abordam exclusivamente os estressores académicos.

Geralmente, entre os estressores gerais, estudo descreve a falta de tempo ou o tempo limitado para realizar as atividades acadêmicas. Os estressores específicos incluem a sobrecarga acadêmica de trabalhos de casa, exames (provas), apresentações de trabalhos, atribuições obrigatórias, exercícios, atividades em sala de aula, a manutenção de um bom desempenho e avaliações pelos professores. Nesse sentido, estudo identificou que $84,4 \%$ dos estudantes de odontologia consideravam o curso de graduação estressante, entre os elementos estressantes incluía a apresentação de trabalhos clínicos em um período curto de tempo e atender os pacientes mais resistentes ${ }^{(24)}$.

A sobrecarga acadêmica representou o maior estressor entre os estudantes de enfermagem, devido ao elevado número de créditos exigido e trabalhos obrigatórios. Assim, muitas vezes a resposta ao estresse tem sido o tabagismo e o consumo de bebidas alcoólicas, a prevalência de uso de álcool é de $50 \%$. No Chile, a prevalência de estresse foi de $36,3 \%$, mais elevada em mulheres $(p<0,05)$. Estudantes de medicina sofrem 
significantemente de mais estresse, especialmente no primeiro ano $(p<0,01)$, quando comparado a estudantes de outros cursos, foi observado baixa prevalência entre os estudantes de Psicologia $(p<0,05)^{(13)}$.

Pode-se concluir que todas as situações produzem diferentes níveis de estresse, relacionadas com o fator de atividade/tempo, com a preocupação por ser a resposta mais comum frente ao estresse ${ }^{(14)}$.

O presente estudo apresenta algumas limitações, em relação aos dados que foram coletados apenas uma escola. A amostra de estudantes pode não ser representativa da população de estudantes usuários de álcool, por ter sido realizada por meio de provas simples de amostragem, desse modo deve ser avaliada com cautela.

\section{Conclusão}

Os estudantes de medicina e tecnologia médica apresentaram níveis elevados de uso álcool no último ano. A predominância do consumo de álcool foi entre jovens do sexo masculino, o uso de álcool está relacionado ao estresse, estressores acadêmicos predispõem ao estresse e são fatores de risco para a utilização de mecanismos negativos, tais como o consumo de álcool. Os resultados têm implicações para repensar como lidar com a prevenção no contexto da Universidade, implementando estratégias que contribuem para minimizar os danos causados pelo álcool na vida destes futuros profissionais.

\section{Referências}

1. World Health Organization. Global status report on alcohol and health. Geneva; 2011.

2. Rehm J, Monteiro M. Alcohol consumption and burden of disease in the Americas: implications for alcohol policy. Rev Panam Salud Pública; 2005 [cited 2016 Mar 10]; 18 (4-5):241-8. Available from: http://www.scielosp.org/ scielo.php?script $=$ sci_arttext\&pid $=$ S10204989200 $5000900003 \&$ Ing $=$ en.http://dx.doi.org/10.1590/ S1020-49892005000900003

3. Comisión Interamericana para el Control del Abuso de Drogas. Organización de los Estados Americanos (CICAD/OEA). I Estudio epidemiológico andino sobre consumo de drogas en la población universitaria. Informe Regional; 2009.

4. Comisión Interamericana para el Control del Abuso de Drogas. Organización de los Estados Americanos (CICAD/OEA). II Estudio epidemiológico andino sobre consumo de drogas en la población universitaria. Informe Regional; 2012.

5. Comisión Nacional para el Desarrollo y Vida sin Drogas. DEVIDA. Reporte Estadístico 2010. Perú; 2010.
6. Organización Mundial de la Salud. Comisión de Determinantes Sociales de la Salud. Subsanar las desigualdades en una generación. Informe Final. 2009. Disponible en: http://www.who.int/social_determinants/ thecommission/finalreport/es/

7. Barraza A. Estrés académico: Un estado de la cuestión. Revista PsicologíaCientífica.com. 2007; 9(2). Disponible en: http://www.psicologiacientifica.com/ estres-academico-2.

8. Salazar E, Ugarte M, Vásquez L, Loaiza J. Consumo de alcohol y drogas y factores psicosociales asociados en adolescentes de Lima. Anales de la Facultad de Medicina UNMSM. [Internet]. 2004; 65(3): Disponible en: http:// revistasinvestigacion.unmsm.edu.pe/index.php/anales/ article/view/1389

9. Gregorio MAPS, Rodríguez AM, Borda M, Río C del. Estrés y rendimiento académico en estudiantes universitarios. Cuad Med Psicosom Psiquiatr Enlace. 2003; (67/68):26-33.

10. Perales A, Sogi C, Morales R. Estudio comparativo de salud mental en estudiantes de medicina de dos universidades estatales peruanas. Anales Fac Med. [Internet]. 2003 [Acceso 20 jun 2016]; 64(4):23946. Disponible en: <http://www.scielo.org.pe/ scielo.php?script $=$ sci_arttext\&pid=S1025-55832003000 400007\&lng $=$ es\&nrm $=$ iso $>$.

11. Whitehead M, Dahlgren G, Gilson L. Developing the policy response to inequities in Health: A global perspective. Challenging inequities in health care: from ethics to action. New York: Oxford University Press; 2001. p. 309-22.

12. Cogollo M, Arrieta V, Blanco B, Ramos L, Zapata K, Rodríguez $Y$. Factores psicosociales asociados al consumo de sustancias en estudiantes de una universidad pública. Rev Salud Pública. [Internet]. 2011 Jun [Acceso 20 jun 2016]; 13(3):470-479. Disponible en: http://www. scielo.org.co/scielo.php?script=sci_arttext\&pid=S012400642011000300009\&lng=es.

13. Marty M, Lavin G, Figueroa M, Larrain C D, Cruz M. Prevalencia de estrés en estudiantes del área de la salud de la Universidad de los Andes y su relación con enfermedades infecciosas. Rev Chil Neuro-Psiquiatr. [Internet]. 2016 Mar [Acceso 20 jun 2016]; 54(1):19-26. Disponible en: http://www. scielo.cl/scielo.php?script=sci_arttext\&pid=S0717$92272016000100003 \&$ Ing=es. http://dx.doi. org/10.4067/S0717-92272016000100003.

14. Phun ET, Santos CB dos. El consumo de alcohol y el estrés entre estudiantes del segundo [Acceso 20 jun 2016]; 18(spe):496-503. Disponible en: http://www. scielo.br/scielo.php?script=sci_arttext\&pid=S0104$11692010000700003 \&$ Ing=en. http://dx.doi. org/10.1590/S0104-11692010000700003. 
15. Babor TF, Higgins-Biddle JC, Saunders, JB, Monteiro MG. Cuestionario de Identificación de los Trastornos debidos al Consumo de Alcohol. Departamento de Salud Mental y Dependencia de Sustancias. Organización Mundial de la Salud. Ginebra; 2001. Disponible en: http://www.who.int/substance_abuse/activities/en/ AUDITmanualSpanish.pdf

16. Monteiro SA, Casado PG, Cruz CL, Fernández FB. Papel del Test AUDIT (Alcohol Use Disorders Identification Test) para la detección de consumo excesivo de alcohol en Atención Primaria. MEDIFAM 2001;11(9):553-7.

17. Roncero C, Egido A, Rodríguez L, Pérez J, Collazos F, Casas $M$. Consumo de drogas entre los estudiantes de medicina: una revisión de la literatura 1988-2013. Actas Esp Psiquiatr. 2015;43(3):109-21.

18. Puig-Nolasco A, Cortaza-Ramirez L, Pillon SC. Consumo de alcohol entre estudiantes mexicanos de medicina. Rev. Latino-Am. Enfermagem. [Internet]. 2008 Aug [Acceso 20 jun 2016]; 16(spe):595-600. Disponible en: http://www.scielo.br/scielo.php?script=sci arttext\&pid $=$ S0104-11692008000700015\&lng =en. http://dx.doi.org/10.1590.

19. Montoya Vásquez EM, Cunningham J, Brands B, Strike C, Wright MGM. Consumo percibido y uso de drogas lícitas e ilícitas en estudiantes universitarios Medellín, Colombia. Rev. Latino-Am. Enfermagem [Internet]. 2009 [Acceso 20 jun 2016]; 17(spe):886-92. Disponible en: http://www.scielo.br/scielo.php?script $=$ sci_ arttext\&pid $=$ S0104-11692009000700020\&lng =en. http://dx.doi.org/10.1590/S0104-11692009000700020. 20. Buchanan J, Pillon SC. Uso de drogas entre estudiantes de medicina, Tegucigalpa, Honduras. Rev. Latino-Am. Enfermagem [Internet]. 2008 Aug [Acesso 20 jun 2016]; 16(spe):595-600. Disponível em: http://www.scielo.br/scielo.php?script $=$ sci_ arttext\&pid $=$ S0104-11692008000700015\&lng $=$ en . http://dx.doi.org/10.1590/S0104-11692008000700015. 21. Masten AS, Faden VB, Zucker RA, Spear LP. A development al perspective on underage alcohol use. Alcohol Res Health. 2009;(32):1-15.

22. Velleman R. Influences on how children and young people learn about and behave towards alcohol: a review of the literature for the Joseph Rowntree Foundation (Past 1). York, UK; 2009.

23. Gomes M. A Monitoreo y análisis de los procesos de cambio de los Sistemas de Salud. Taller San Juan, Puerto
Rico. Agosto 2005. Panel 1: Contexto del Sistema de Salud. Presentación: Determinantes de la Salud. OPS/ OMS. Nicaragua: OPS; 2005.

24. Vargas YG. Estrés en el estudiante de odontología. Ponencia presentada en el Primer Foro de Experiencias PAEA. [Internet]. 2001 [Acceso 20 jun 2016]; Disponible en: http://www.iztacala.unam.mx/temas/ foropaea/29RCD07Ia.htm. 